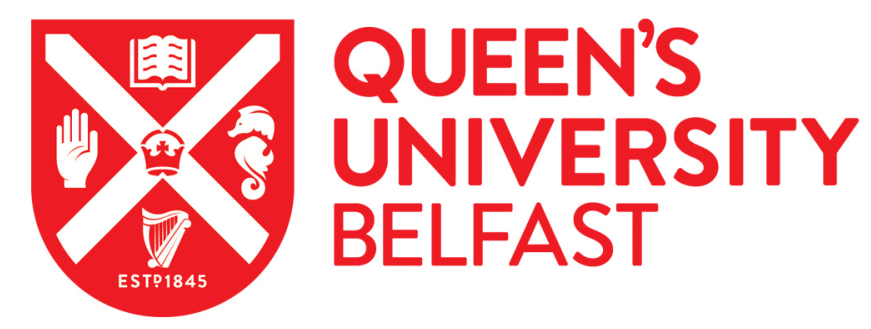

\title{
Pilot Tone Actuated Antenna Array Pattern Reconfiguration
}

Fusco, V., Zelenchuk, D. E., \& Buchanan, N. B. (2014). Pilot Tone Actuated Antenna Array Pattern Reconfiguration. IEEE Transactions on Antennas and Propagation, 62(2), 629-640.

https://doi.org/10.1109/TAP.2013.2290292

\section{Published in:}

IEEE Transactions on Antennas and Propagation

\section{Document Version:}

Peer reviewed version

Queen's University Belfast - Research Portal:

Link to publication record in Queen's University Belfast Research Portal

\section{General rights}

Copyright for the publications made accessible via the Queen's University Belfast Research Portal is retained by the author(s) and / or other copyright owners and it is a condition of accessing these publications that users recognise and abide by the legal requirements associated with these rights.

Take down policy

The Research Portal is Queen's institutional repository that provides access to Queen's research output. Every effort has been made to ensure that content in the Research Portal does not infringe any person's rights, or applicable UK laws. If you discover content in the Research Portal that you believe breaches copyright or violates any law, please contact openaccess@qub.ac.uk. 


\title{
Pilot Tone Actuated Antenna Array Pattern Reconfiguration
}

\author{
V. Fusco, Fellow, IEEE, D. Zelenchuk, Member, IEEE, and N. Buchanan
}

\begin{abstract}
A new strategy for remote reconfiguration of an antenna array far field radiation pattern is described. The scheme uses a pilot tone co-transmitted with a carrier signal from a location distant from that of a receive antenna array whose far field pattern is to be reconfigured. By mixing the co-transmitted signals locally at each antenna element in the array an IF signal is formed which defines an equivalent array spacing that can be made variable by tuning the frequency of the pilot tone with respect to the RF carrier. This makes the antenna array factor hence far field spatial characteristic reconfigurable on receive. For a 10x1 microstrip patch element array we show that the receive pattern can be made to vary from 35 to 10 degrees half power beam width as the difference frequency between the pilot and the carrier at $2.45 \mathrm{GHz}$ varies between $10 \mathrm{MHz}$ and $500 \mathrm{MHz}$ carrier.
\end{abstract}

Index Terms - antenna array, far-field pattern control, mixing, IF beam-steering, self-focussing

\section{INTRODUCTION}

A NTENNA arrays possessing the capacity to have their far field radiation characteristics made reconfigurable has been the subject of intensive research. The requirement to be able to reconfigure far field pattern is desirable in a wide variety of application scenarios. Example applications where this facility could be useful include; RFID [1], sensor networks [2], ad hoc wireless networks [3], radar object tracking [4], satellite communications [5]. A variety of techniques have been developed to allow pattern reconfiguration, notably phased array technology [6]. Ultimately phased array methods rely on the introduction of a phase coherent reference oscillator and individual phase control elements applied locally at each of the separate RF paths comprising the antenna array. Generally this followed by a signal combining stage in order for the antenna array factor to be formed. The classical approach is to modify the phase and, also in some cases, the amplitude weights across the array so that pattern reconfiguration can be effected. Other approaches where less precise control of the spatial characteristics of the array is required can be predicated upon the use of switchable loading elements introduced into the array, many examples of this are available e.g. $[7,8]$. Here the

Manuscript received May 22, 2013. This work was sponsored by the UK Engineering and Physical Science Research Council, grant EP/J013897/1.

The authors are with ECIT Research Institute School of Electronic, Electrical Engineering and Computer Science Queens University of Belfast (e-mail: v.fusco@ee.qub.ac.uk). means for pattern control relies on direct perturbation of near field currents due to structural or electrical modification hence far field pattern control.

With conventional phased array approaches phase reference distribution issues persist. Additionally element spacing in these arrays has to be compliant with grating lobe spacing requirements as dictated by the RF carrier wavelength.

Pilot tones are routinely used in satellite systems for identification and for calibration purposes, [9]. In [10] a less obvious use of a pilot tone was articulated. Here a pilot tone was sent from a remote location and its frequency was selected to be almost identical to that of the RF carrier also transmitted from the same remote location. Local mixing of these signals on a per array element basis meant that, irrespective of the angle of arrival, the received signal phases after down-mixing were in phase. This means that the array is capable of optimally recombining the received RF signals arriving from any direction, in effect it is self-focussing.

In this paper we describe an extension to this special case whereby the far field pattern characteristics of an antenna array operating in receive mode can be controlled from a remote location by means of a pilot tone, co-transmitted along with the RF carrier signal. This approach enables agile receive pattern re-shaping and also permits IF beam steering. In addition it preserves the important special case of a receive array that can self-focus onto an incoming signal whose direction is not known a-priori. The resulting configuration would be useful in applications were the field of view of the antenna can be programmed remotely, for example, from a UAV or satellite, then IF beam steered for scene surveillance.

Section II of the paper describes the theory underpinning the approach. Section III illustrates how the properties of such a system can be assessed. Section IV discusses the core analogue signal processing necessary for practical implementation and provides experimental confirmation of the theoretical proposition. Section $\mathrm{V}$ draws conclusions and identifies some aspects that would merit further study.

\section{PRINCIPLE OF OPERATION}

With reference to Fig.1a the angle of arrival of the pilot tone and the RF carrier at the array is $\theta$, consequently across an $\mathrm{N}$ element array the overall delay will be $\mathrm{N} \psi$ where $\psi$ is defined below.

Let $\cos \left(\omega_{p} t+\phi\right)$ be a pilot tone, and let $A(t) \cos \left(\omega_{c} t+\phi\right)$ be an amplitude encoded RF carrier, each have instantaneous phase $\phi$. From Fig.1 after mixing and suitable filtering at the 
${ }^{\text {th }}$ element the signal output $E_{k}$ is for

where

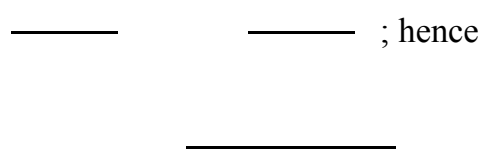

If

then the term containing angle of arrival

information goes to zero, hence all are in phase, and the array is self-focusing at the carrier frequency occurs.

We can rewrite (1) as

where

Equation (2) shows that we have the conditions for a receiving antenna array that is operating in a manner equivalent to an antenna array beam forming at the intermediate frequency, IF, without the need for a phase matched coherent master reference signal locally applied at each array element through a corporate feed distribution network. An additional feature of the remote pilot arrangement is that while the receive elements operate at the RF frequency (wavelength small) the array spacing requirement dictated by the IF frequency (wavelength large) determines the antenna array factor. This is obtained as the superposition of the outputs of all $E_{k}$ elements across the array. Hence the array factor is delivered at the appropriate IF wavelength and the grating lobe RF imposed element separation criteria do not apply. Therefore the array element spacing can be many wavelengths at the RF frequency, i.e. the array can be made very sparse, without violating gating lobe restrictions imposed by RF frequency spacing requirement. This is a very useful feature for applications where elements may be wavelengths separated from each other.

Inspection of (2) indicates that the receive array pattern can be made agile by virtue of changing the pilot tone frequency with respect to the carrier frequency in effect providing a simple means for changing the $\mathrm{d} / \lambda$ array spacing without changing the physical array element spacing $d$ shown in Fig.1a. Fig.1b shows conceptual detail of the branching filter used to provide RF carrier and pilot tone separation followed by IF signal formation, see also the description in section IV.

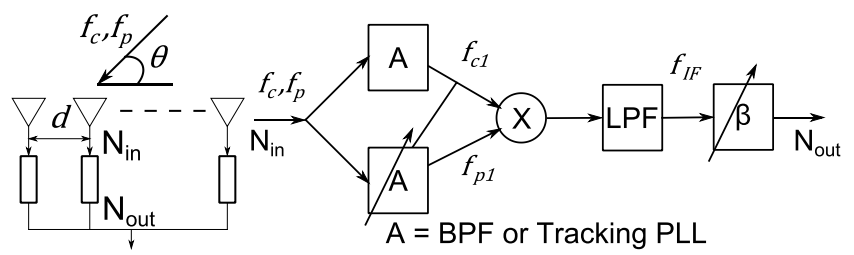

(a)

(b)

Fig.1 Pilot tone actuated reconfigurable antenna array: (a) - the array, (b) array element.

\section{PROPERTY ASSESSMENT}

Let us now consider a typical requirement arrangement for operation in the $2.45 \mathrm{GHz}$ (vii) Industrial, Scientific, and Medical, ISM, band, [11], where the available bandwidth is $\pm 50 \mathrm{MHz}$, Fig. 2 .

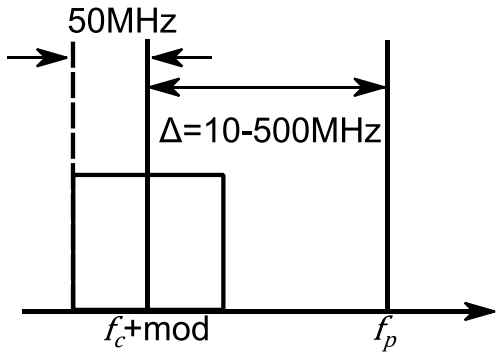

Fig.2 Example of frequency spacing

Typical values for pilot offset with $2.4 \mathrm{GHz}$ carrier frequency for this application are given in Table 1 under the array conditions stipulated below;

$$
\text { If we set then }=0.6 \mathrm{~m} \text { and }
$$

which we select this as the fixed base separation for the array. Hence as $f_{I F}$ is varied also varies, Table I.

$$
\begin{array}{cc}
\text { TABLE I } & \\
\text { VARIATION OF } & \text { WITH IF FREQUENCY ASSUMING } \\
\text { MHz, } \mathrm{f}_{\mathrm{p}}=2.4 \mathrm{GHz} . & \text { AT } 500
\end{array}
$$

\begin{tabular}{ccc}
\hline \hline & & HPBW(deg) $10 \times 1$ array \\
\hline 250 & 0.25 & 25 \\
100 & 0.1 & 52 \\
50 & 0.05 & half space
\end{tabular}

Consequently the apparent electrical spacing between array elements separated by $\mathrm{d}$ appears to change as $f_{I F}$ is varied. Hence by varying the pilot tone frequency we have an electrical method for changing array factor without mechanically re-configuring it.

If we assume that the total average transmitted power in the system is $P$, and is comprised of the information bearing signal power, , bandwidth $B_{c}$, and the pilot signal power , bandwidth $B_{p}$. Both of these signals traverse the same channel and since they are co-located at the point of transmission we can approximate that the channel noise environment they experience is the same. Therefore under the assumption that at the receive array elements the signal to noise ratio of pilot and carrier signals are the same $S / N_{p}=S / N_{c}$, then hence

Equation (3) can be used to set the relative pilot to carrier power levels once the carrier bandwidth is assigned.

\section{CHARACTERISATION}

Using the architecture in Fig.1b we see that in order for such a system to operate with a prescribed bandwidth RF 
signal that extraction of the pilot tone will require a narrowband filter whose band centre is tuneable, Fig. 3a. In Fig.1b, Fig.3b the separated signals are then combined by mixing to extract the IF frequency and the preserved relative phase between carrier and pilot needed for pattern reconfiguration as defined by (2). Summing mixer outputs across all of the elements in the array allows the IF array factor to be formed. In a practical scenario data embedded within the down converted carrier signal fcl could be used to program the divider ratio of the pilot tone tracking PLL centre frequency to the value required for the particular pattern to be configured, shown in Fig.3a as the BPF to PLL cross link.

In order to test the viability of extracting the relative phase difference between co-transmitted pilot and carrier directly in the RF frequency region the experimental configuration shown in Fig. $3 b$ was used. Here for experimental convenience the pilot tone was fixed at $2.4 \mathrm{GHz} @-54 \mathrm{dBm}$, while the centre frequency of the carrier was allowed to vary from 1.9 to $2.39 \mathrm{GHz}$ at a constant power level of $-24 \mathrm{dBm}$. The tracking PLL capture range was $+/-12 \mathrm{KHz}$ at $2.4 \mathrm{GHz}$, this narrow capture range allows the pilot to be reliably recovered down to very low signals levels. The difference in power between the pilot signal and RF signal can be selected on the basis of (3), we choose the ratio to be $36 \mathrm{~dB}$ as being representative of a real system. This has the additional benefit that no filtering is needed in the RF chain. Further, the use of a low level pilot signal minimises the possibility of mixer intermodulation products forming at the receive side, again simplifying filtering arrangements. The three sources in Fig. $3 \mathrm{~b}$ are phase locked to a single $10 \mathrm{MHz}$ reference so that absolute phase measurements can be made using the oscilloscope. A double balanced mixer type HP IAM-81008 was used.

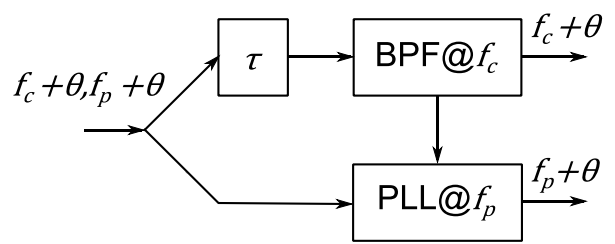

(a)

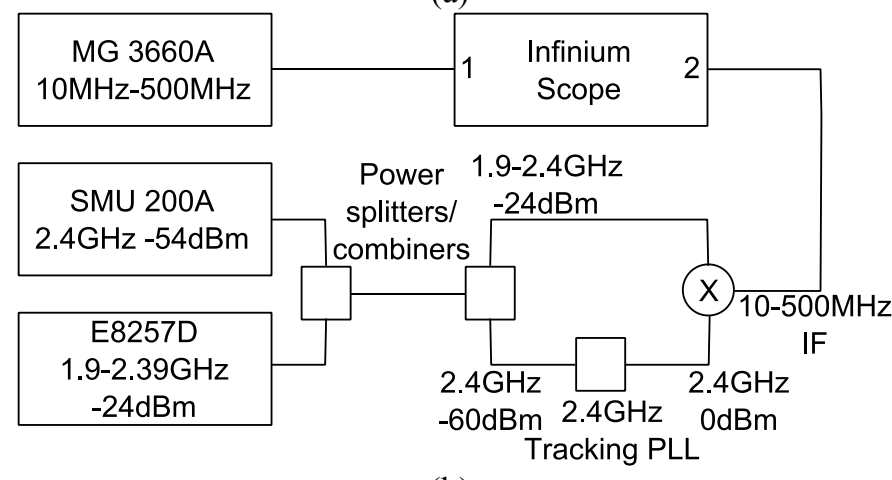

(b)

Fig.3 Experimental arrangement: (a) conceptual, (b) actual

In Fig.3a delay $\tau$, is included to illustrate that in an actual system delay could result due to branch path cable length differences, filter group delays, etc. In the experimental setup in Fig. 3b, we avoid the need for $\tau$, through a simple calibration process. At each IF the resulting offset phase due to the instantaneous start-up phase of the reference phase locking oscillator was subtracted from all subsequent phase readings for the particular IF under consideration, i.e. $\tau$ is set to 0. Fig.4 plots the phase retrieved at the mixer output versus delay length. These results show that for each IF case nearly linear IF phase responses can be extracted directly at RF under realistic input signal amplitude conditions.

The input variable, 'delay length', represents the delay that both pilot and carrier experience at the $\mathrm{k}^{\text {th }}$ array element due to a signal arriving at angle $\theta$. Delay length is used in Fig.4 so that plots for different IFs can be overlaid onto a single $\mathrm{x}$-axis, e.g. a $100 \mathrm{MHz}$ IF is equivalent to $3 \mathrm{~m}$ wavelength, so for a linear down-conversion transfer function $1 \mathrm{~m}$ of delay length should yield $120^{\circ}$ of output phase shift.

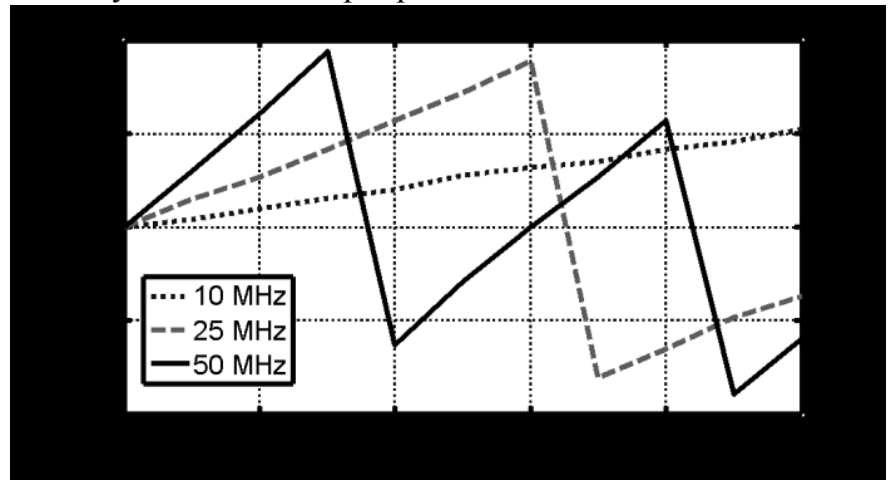

(a)

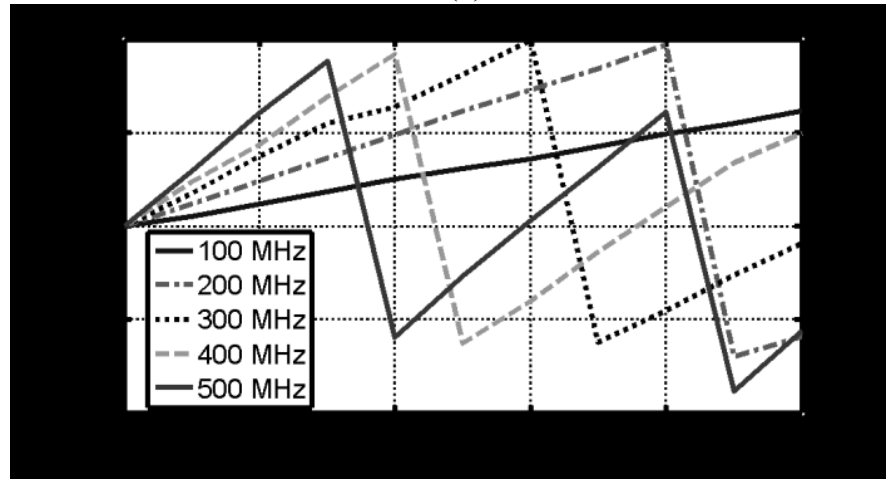

(b)

Fig.4 Measured mixer phase transfer function.

When pilot and carrier signals at the same angle of incidence with a phase delay between them fall onto the antenna array the intermediate frequency is recovered with some minor distortion, i.e.

The phase delay , ideally obtained through measurement is shown in Fig .4, this data, after phase unwrapping, is used to compute the IF array factor, which for a $1 \mathrm{D}$ array is

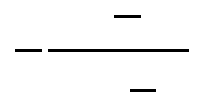

here , with - -velocity of 
light, -scanning angle, -phase difference between the antenna elements separated by distance. The separation between antennas is taken as $=0.3 \mathrm{~m}$ and the number of elements is

Since no additional phase shift is applied at the beam forming network i.e. , then As with conventional IF beam steering if the IF array pattern can be steered. Since beam forming is occurring at the IF the physical means for realising these phase shifters is much simpler than it would be at RF. The array factor calculated using the measured recovered IF phase responses are plotted in Fig.5. Minor deviation of ideal array performance with respect to measured performance is mainly due to mixer impedance mismatch on the LO $2.4 \mathrm{GHz}$ port. In Fig. 6 the resulting IF patterns obtained using a $10 \times 1$ array of $0.3 \mathrm{~m}$ spaced $2.4 \mathrm{GHz}$ resonant microstrip patch elements is given. Overlaid on these is the response of the array when operated by directly beam forming at RF, here the effect of grating lobes due to large element separation at RF is clearly visible. It should be noted that since the array is sparse at the RF carrier frequency, $\mathrm{d} \sim 2.5 \lambda$ isolated antenna element array pattern characteristics apply. These results show that as $\mathrm{d} / \lambda_{I F}$ increases towards 0.5 that the directivity of the pattern increases, and that only a single main beam is formed. It is also interesting to note that when $d=0 \lambda$ the resultant array factor is unity as the phase centres of each array element are coincident, this is the self-focussing case.

Hence for a fixed physical array separation $d$ we have a practical method by which the $\mathrm{d} / \lambda_{I F}$ ratio of the array can be recovered and used according to the theory presented in Section I for the purposes of array pattern reconfiguration.

It is also of interest to note that due to the low frequencies associated with the IF beam forming strategy suggested here that data at array elements can be captured and memorised by digital means. This opens a wide range of possibilities for pattern manipulation through simple post processing. For example in Fig. 7 we show the consequences of multiplying the recorded IF patterns together. This has the net effect of slightly reducing main lobe half power beam width while simultaneously reducing sidelobe levels to below $-30 \mathrm{~dB}$ for incident angles $>75^{\circ}, 90^{\circ}=$ boresight.

\section{CONCLUSION}

We have shown a scheme based on co-transmitted pilot and $\mathrm{RF}$ carrier wherein local mixing on a per element basis can be used to control the antenna receive array factor, hence its receive spatial pattern. Consequently spatial array patterns are remotely controlled by tuning the frequency of the pilot signal. The ability to control the antenna array receive characteristic in a simple manner should prove useful in a range of sensor and re-configurable wireless local area network beam forming applications which were previously intractable, or extremely difficult to implement, due to array element LO phase synchronisation issues.
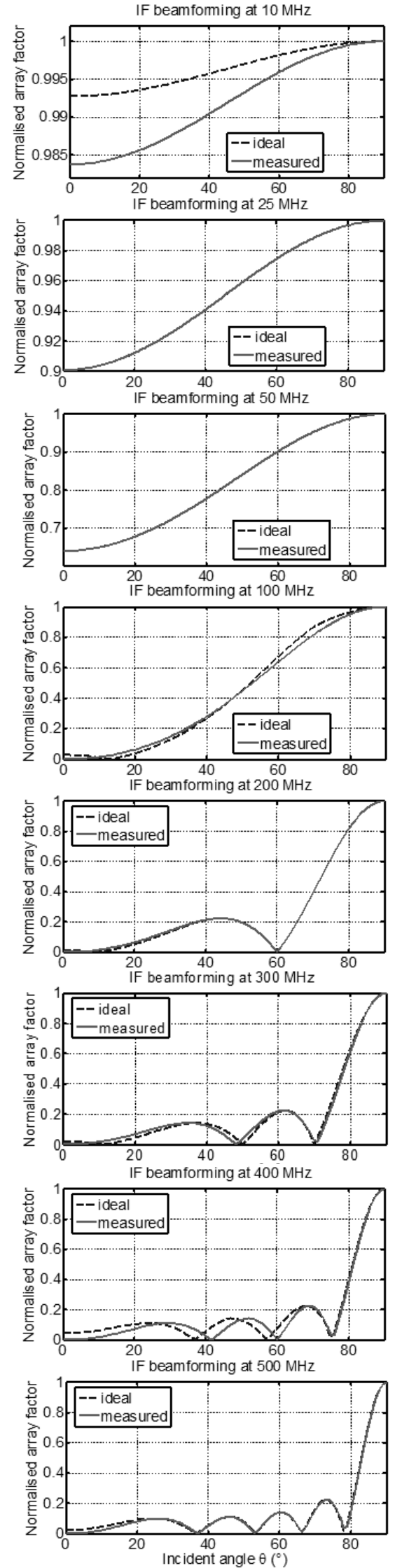

Fig. 5 Comparison of array factor at different intermediate frequencies for ideal and measured recovered phase between pilot and carrier for 10x1 array with $\mathrm{d}=0.3 \mathrm{~m}, 90^{\circ}=$ boresight. 


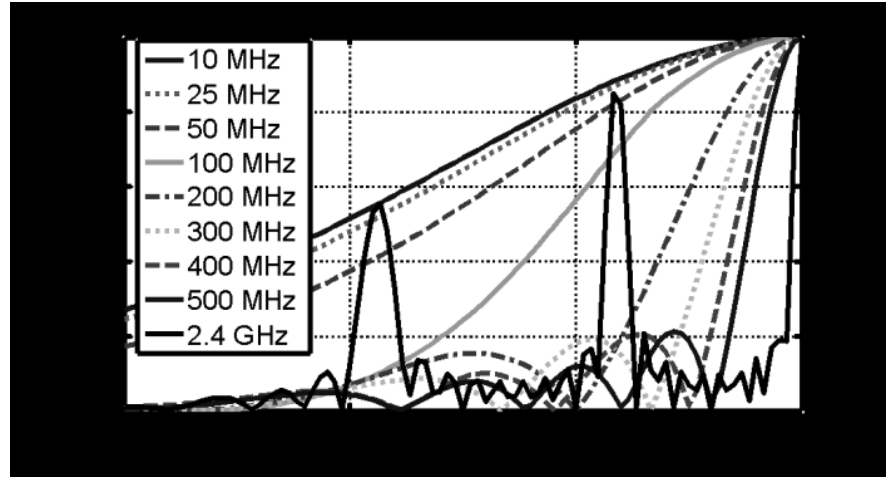

Fig. 6 IF patterns obtained using a $10 \mathrm{x} 1$ array of $0.3 \mathrm{~m}$ spaced $2.4 \mathrm{GHz}$ resonant microstrip patch elements; $-2.4 \mathrm{GHz}$ indicates response of array directly formed at RF.

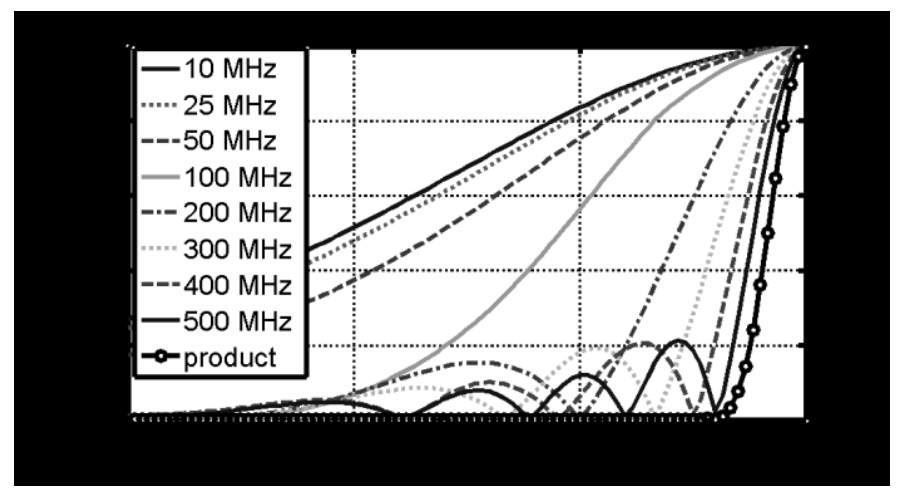

Fig.7 Sidelobe suppression using multiple IF pattern multiplication for 10x1 array of $0.3 \mathrm{~m}$ spaced $2.4 \mathrm{GHz}$ resonant microstrip patch elements

\section{REFERENCES}

[1] H. Rhyu, F. J. Harackiewicz, B. Lee, "Wide coverage area of uhf-band RFID system using a pattern reconfigurable antenna", Microwave and Optical Technology Letters, vol. 49, no. 9, Sept. 2007, pp. 2154-2157.

[2] Y. Loke, M. Tong, E.C. Yeo, R. Broadston,"Distributed phased arrays with wireless beamforming", Forty-First Asilomar Conference on Signals, Systems and Computers, Nov. 2007, pp. 948-952.

[3] J. Kountouriotis, D. Piazza, P. Mookiah, K.R. Dandekar, "Reconfigurable antennas and configuration selection methods for mimo ad-hoc networks", EURASIP Journal on Wireless Communications and Networking (Special Issue on Recent Advances in Multiuser MIMO Systems), 2011, 2011:147.

[4] M.I. Skolnik, Introduction to Radar Systems, McGraw-Hill International Editions: Electrical Engineering Series, 3rd Edition, 1997.

[5] S.K. Rao, "Reconfigurable Antenna System for Satellite Communications", 2007 IEEE Antennas and Propagation Society International Symposium, June 2007, Hawaii, pp. 3157-3160.

[6] R. Mailloux, Phased Array Antenna Handbook, Artech House Antenna Library, 2nd Edition 2005.

[7] J.A. Rodríguez, "Beam reconfiguration in antenna arrays by using parasitic elements", 2007 IEEE Antennas and Propagation Society International Symposium, June 2007, Hawaii, pp. 3141-3144.

[8] F. Venneri, "Design of a reconfigurable reflectarray based on a varactor tuned element", 2012 6th European Conference on Antennas and Propagation, March 2012, pp. 2628-2631.

[9] I. Korn, Coherent detection of M-ary phase-shift keying in the satellite mobile channel with tone calibration, IEEE Trans. on Communications, Vol. 37, No. 10, pp907-1003, Oct 1989.

[10] V.Fusco, Q.Chen, H.Gamble, B. Armstrong, "Self-steered Silicon Receiver Array", Silicon Monolithic Integrated Circuits in RF Systems, 2000 IEEE Topical Meeting, Garmish, pp $25-28$.

[11] ISM band,

http://www.princeton.edu/ achaney/tmve/wiki100k/docs/ISM_band.htm 1, last accessed March 2013.

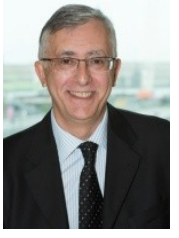

Vincent Fusco holds a personal chair in High Frequency Electronic Engineering at Queens University Belfast.

His research interests include active antenna and front-end MMIC techniques. He is head of the High Frequency Laboratories at QUB where he is also director of the International Centre for System on Chip for Advanced Microwireless.

He has published over 450 scientific papers in major journals and in referred international conferences. He has authored two text books, holds patents related to self-tracking antennas and has contributed invited papers and book chapters. He serves on the technical program committee for various international conferences including the European Microwave Conference.

He is a Fellow of both the Institution of Engineering and Technology and the Institute of Electrical and Electronic Engineers. In addition he is a Fellow of the Royal Academy of Engineers and a member of the Royal Irish Academy.

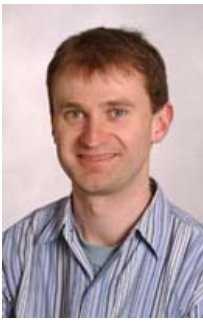

Neil B. Buchanan received a B.Eng. Hons degree from the Queens University of Belfast in 1993. In July 2000, he graduated with a Ph.D. entitled "Phase Locked Millimetre Wave HEMT Oscillators" from the Queens University of Belfast and is currently employed as a Lecturer within the High Frequency Electronics Group at that university. His research interests include self-steered (retrodirective) antennas, super regenerative detectors, injection-locked oscillators, and mm-wave MMIC design. He has published over 50 papers in refereed journals and conferences and has provided consultancy to a number of companies. He has acted as technical manager for a number of research/industrial projects and has refereed IEE/IEEE journals and conferences.

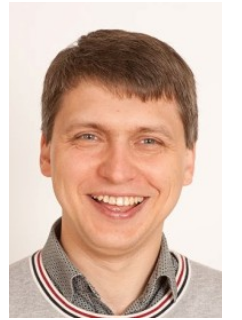

Dmitry E. Zelenchuk (S'02, M'05) received his $\mathrm{PhD}$ in Radiophysics from Rostov State University (Russia) in 2004.

From 2003 till 2005 he was a Lecturer at the Dept. of Applied Electrodynamics and Computer Modelling, Rostov State University, Russia. Currently he is Senior Engineer at Queen's University Belfast, UK. His research interests include electromagnetic field theory, material characterization; mm-wave circuits, antennas and advanced mm-wave packaging; propagation in complex environments; and various physical phenomena of plasmonic and nanostructures. He has published more than 70 journal and conference papers and a book chapter and been a session chair at scientific conferences.

In 2001 Dr. Zelenchuk was awarded the medal of Ministry of Education of the Russian Federation "For the Best Scientific Student Paper". 